\section{$\underset{\substack{\text { hommes } \\ \text { \& migrations }}}{ }$}

\section{Hommes \& migrations}

Revue française de référence sur les dynamiques

migratoires

$1301 \mid 2013$

Migrations et mondes ruraux

\title{
Les ouvriers saisonniers kurdes
}

Travaillant à la cueillette des noisettes en Turquie

\section{Mustafa Aslan}

\section{(2) OpenEdition}

1 Journals

\section{Édition électronique}

URL : http://journals.openedition.org/hommesmigrations/1972

DOI : 10.4000/hommesmigrations. 1972

ISSN : 2262-3353

Éditeur

Musée national de l'histoire de l'immigration

\section{Édition imprimée}

Date de publication : 1 janvier 2013

Pagination : 129-136

ISBN : 978-2-919040-21-6

ISSN : 1142-852X

Référence électronique

Mustafa Aslan, « Les ouvriers saisonniers kurdes », Hommes \& migrations [En ligne], 1301 | 2013, mis en ligne le 31 décembre 2015, consulté le 01 mai 2019. URL : http://journals.openedition.org/ hommesmigrations/1972; DOI : 10.4000/hommesmigrations.1972 


\title{
LES OUVRIERS \\ SAISONNIERS KURDES \\ TRAVAILLANT À LA CUEILLETTE DES NOISETTES EN TURQUIE
}

par MUSTAFA ASLAN, maître de conférences, anthropologue à l'université de Siirt (Turquie)

\author{
À l'ouest de la Turquie, dans les provinces de Düzce \\ et de Sakarya, la saison des noisettes marque l'embauche \\ de nombreux ouvriers saisonniers kurdes. Ces ramasseurs \\ de noisettes, jeunes et démunis pour la plupart, sont à \\ la merci des intermédiaires qui organisent leur recrutement. \\ Ils sont contraints de vivre dans des logements insalubres et, \\ pour une activité épuisante, ne reçoivent qu'un salaire dérisoire. \\ Ils subissent à la fois la politique de répression pratiquée \\ en Turquie contre la minorité kurde et la concurrence du marché \\ agro-alimentaire international.
}

\section{On appelle généralement "travailleurs saisonniers agricoles" tous ceux qui effectuent des travaux agricoles ainsi que la cueillette des fruits et légumes.}

Le nombre de travailleurs saisonniers employés dans l'agriculture turque est de l'ordre d'un million d'hommes, de femmes et d'enfants ${ }^{1}$. Parmi eux, de nombreux saisonniers kurdes se rendent tous les ans, à partir du mois de février, dans les régions de la mer Égée, de la Méditerranée, de l’Anatolie centrale et de la mer Noire pour y effectuer divers travaux agricoles dans des conditions extrêmement difficiles. En Turquie, il n'existe pas une définition précise et opératoire du "travailleur saisonnier". On appelle généralement "travailleurs saisonniers agricoles" tous ceux qui effectuent des travaux agricoles ainsi que la cueillette des fruits et légumes. Selon l'Institut des statistiques de Turquie (TUIK), le travailleur saisonnier est celui qui "ne travaille pas régulièrement ou en permanence et qui est embauché une ou plusieurs fois pendant l'année agricole".

Cette analyse s'appuie notamment sur des données collectées lors des enquêtes de terrain réalisées durant les étés 2009 et 2010 dans les provinces de Düzce et de Sakarya (situées à 150 km à l'est d'Istanbul, sur la côte ouest de la mer Noire). Dans le cadre de ces enquêtes, plusieurs interviews ont été réalisées avec des conducteurs de dolmus (des minibus de 14 places), avec des intermédiaires qui re- 
crutent des travailleurs pour les agriculteurs, des chefs d'équipe et des travailleurs eux-mêmes. Une partie de ces interviews ont eu lieu avant le départ ou lors du départ des travail-
Comme partout dans

le monde, en Turquie,

les travailleurs saisonniers

constituent un élément

clé pour le développement de l'agriculture intensive. leurs en 2009. D'autres entretiens ont été menés pendant la saison de la cueillette des noisettes en 2010. Les interviews sur les conditions de voyage, de travail, de logement, sur l'alimentation, les salaires et la du- rée du travail ont été associées à des observations directes. En plus, des entretiens menés auprès des producteurs de noisettes à propos de leur coût de production, des relations entre les agriculteurs et les travailleurs, des recherches d'archives ont permis d'analyser le développement de la culture des noisettes et le sous-développement de la région du sud-est de la Turquie dont sont originaires les saisonniers agricoles kurdes.

\section{Les saisonniers face aux contraintes de la culture intensive des noisettes en Turquie}

Selon les estimations de l'International Labor Organisation (ILO), "450 millions d'hommes et de femmes travaillent comme salariés dans l'agriculture de par le monde. Si l'on y ajoute les agriculteurs propriétaires de leurs terres et les millions de travailleurs de l'économie agricole informelle, ce secteur compte 1,3 milliard de personnes, soit la moitié de la main-d'œuvre mondiale $^{2 "}$. Comme partout dans le monde, en Turquie, les travailleurs saisonniers constituent un élément clé pour le développement de l'agriculture intensive. C'est la main-d'œuvre saisonnière, ajustable par rapport aux fluctuations du marché des fruits et des légumes, qui est la plus intéressante pour les exploitants de l'agriculture intensive. "En raison des concurrences internationales acharnées qui pèsent sur les producteurs, le salaire constitue la seule variable d'ajustement en termes de coûts.". Cela est également valable pour la Turquie, où la maind'œuvre constitue $50 \%$ du coût de la production des noisettes.

La situation des travailleurs saisonniers agricoles n'est ni nouvelle en Turquie, ni spécifique à ce pays. Elle a été largement étudiée ailleurs, par exemple aux États-Unis, au Brésil, en France, en Italie et en Espagne. Toutefois, alors qu'en Europe ou aux États-Unis les travailleurs agricoles saisonniers employés sont pour la plupart originaires d'un autre pays (Mexicains travaillant en Californie, Marocains ou Maliens en France ; Ukrainiens en Espagne), en Turquie les travailleurs saisonniers agricoles sont quasiment tous des nationaux d'origine kurde. Originaires de provinces ou de centres comme Batman, Diyarbakir, Mardin, Siirt, Urfa et Sirnak, les travailleurs saisonniers agricoles kurdes sont embauchés par les producteurs de noisettes. Comme on peut le remarquer sur le tableau ci-dessous, plus des trois quarts de ces travailleurs sont des jeunes âgés entre 16 et 30 ans. Par exemple, sur 110 travailleurs du groupe A, 89 personnes (80,9 \%) ont entre 16 et 35 ans. De même, sur 359 travailleurs composant le groupe B, 285 personnes $(79,4 \%)$ ont entre 16 et 30 ans. Ce qui montre qu'à part les chefs d'équipe et les cuisinières, ce sont surtout des jeunes qui travaillent dans la cueillette des noisettes.

Durant les trois dernières décennies, l'agriculture turque s'est considérablement développée grâce à l'usage des serres, pour lesquelles l'État a octroyé des aides financières ${ }^{4}$. En 2009, les produits agricoles ont constitué 8,3\% du PNB turc ${ }^{5}$. Conséquence de cette augmentation de la production, la Turquie exporte, outre des noisettes, plusieurs variétés de fruits frais (melons, pastèques, oranges, 
La plupart des travailleurs saisonniers kurdes effectuent le voyage soit en car, soit en minibus.

Après plus de 18 heures de voyage continu, le minibus parti du département de Batman vient d'arriver à sa destination, Ortakoy, une commune du département de Sakarya, août 2010. (c) Mustafa Aslan

pommes, clémentines) et secs (figues, raisins et abricots) et de légumes (tomates, aubergines, poivrons, salades, courgettes, concombres).

La production de noisettes s'est développée grâce, à la fois, à un climat propice, à l'octroi de subventions et à la garantie d'achat de noisettes par l'État ${ }^{6}$. À l'heure actuelle, elle représente entre 70 et $75 \%$ de la production mondiale de noisettes ${ }^{7}$. La quasitotalité de la main-d'œuvre saisonnière employée vient de la région de l'est de la Turquie, dont le sous-développement continue d’animer le débat politique. Malgré plusieurs plans économiques quinquennaux (dont le dernier était annoncé en 2008) lancés dans le cadre d'un projet d'aménagement (Güneydoğu Anadolu Projesi, GAP), la région reste sous-développée. Car, ce projet, "prévu pour le développement de la région de l'Est, est en effet un projet national stratégique pour la production de l'énergie. Or, en théorie, il devrait contribuer au développement industriel et à la création des emplois dans la région. Pourtant, en 2008, seulement $15 \%$ des canaux d'irrigation, prévus dans le cadre du projet GAP, sont construits, alors que $74 \%$ de la tranche du projet de la construction des barrages hydrauliques d'énergie est terminée $e^{8 ”}$. 
Rappelons qu'après le déclenchement du conflit armé entre le Parti des travailleurs du Kurdistan (PKK) et les forces armées turques, qui a provoqué entre autres une migration forcée massive ${ }^{9}$, les conditions économiques de la région se sont encore aggravées. Suite à ce conflit, l'état d'urgence a été instauré dans cette région

À partir des années 1970,

ces saisonniers se sont progressivement installés

avec leurs familles dans les villes où ils travaillaient.

Cette installation définitive a fait baisser, dans un premier temps, le nombre de travailleurs saisonniers kurdes.

tanbul, Bursa et Izmir, avec l'espoir d'y trouver du travail. Par exemple, selon les données démographiques de 2009, "sur 18,5 millions d'habitants de la région de l'Est, 7,5 millions de personnes (soit 40,5\%) vivent dans les régions du sud, du centre et de l'ouest de l'Anatolie ${ }^{11 "}$.

\section{La main-d'œuvre kurde dans l'agriculture turque et sa migration vers l'ouest du pays}

En Turquie comme partout ailleurs, l'agriculture intensive a besoin d'une main-d'œuvre disponible au moment voulu, car la récolte ne peut pas attendre. Par exemple, dans le cas des noisettes qui sont cultivées dans des zones pluvieuses, il faut les ramasser le plus vite possible. S'il pleut le moment venu, les noisettes moisiront et l'agriculteur perdra toute sa récolte. C'est pourquoi il a besoin de beaucoup de main-d'œuvre pendant une période courte qui se compte en semaines, voire en jours.
La main-d'œuvre kurde, abondante dans la région du Sud-Est, satisfait ce besoin pressant des agriculteurs turcs. Il s'agit aussi d'une main-d'œuvre disponible et non qualifiée, payée en dessous du Smic turc ${ }^{12}$.

La migration des saisonniers kurdes vers l'ouest de la Turquie existe depuis les années 1960 où les travailleurs originaires de Urfa, Mardin, Diyarbakır, Adıyaman, Siirt ont commencé à migrer vers les régions de l'Égée et de la Méditerranée. Au départ, ils étaient en partie recrutés par les producteurs de coton dans la région de Çukurova (Cilicie) et par les horticulteurs égéens. Les autres travailleurs, pour la plupart des hommes partant seuls, étaient employés dans le secteur de la construction. Dans les deux cas, une fois terminée la saison de la cueillette, les travailleurs rentraient chez eux pour $y$ passer l'hiver ou s'occuper de leur lopin de terre.

À partir des années 1970, ces saisonniers se sont progressivement installés avec leurs familles dans les villes où ils travaillaient. Cette installation définitive a fait baisser dans un premier temps le nombre de travailleurs saisonniers kurdes. Mais ce nombre a beaucoup augmenté après le conflit armé déclenché par le PKK en 1984 contre l'armée turque, qui a forcé les paysans kurdes à quitter leurs villages et à s'installer dans les villes. "Entre 1989 et 1999, 3438 villages et hameaux ont été vidés et entre 4 et 4,5 millions d'individus ont été obligés de quitter leur lieu de résidence ${ }^{13}$." Ces migrants, pour la plupart d'origine paysanne, sont devenus des chômeurs sans profession dans les villes où ils ont émigré. Ils ne parvenaient à se faire recruter que pour des travaux manuels ou saisonniers proposés par les secteurs du bâtiment et de l'agriculture, ceux qui emploient massivement de la maind'œuvre non qualifiée.

Pour le secteur agricole qui offre plus d'opportunités d'emploi que celui du bâtiment et qui avait déjà commencé à manquer de bras, en raison du 
"recrutement de la main-d'œuvre locale par d'autres secteurs ${ }^{14}$, l'arrivée de ces gens non qualifiés sur le marché du travail a été une aubaine. Les producteurs de noisettes de la province de Sakarya employaient, jusqu'aux années 1980, des travailleurs turcs provenant de la région voisine de Balikesir. Mais ceux-ci ayant eu d'autres opportunités de travail, la place était libre pour les saisonniers kurdes ${ }^{15}$. Leur embauche a été plus avantageuse que celle des travailleurs turcs car les Kurdes ont accepté d'être moins payés, de prendre en charge leurs frais de déplacement et de faire plus que les huit heures de travail par jour assurées par les saisonniers turcs.

\section{Recrutement des travailleurs et relations employés/employeurs}

Le recrutement des travailleurs se fait par l'intermédiaire des elçi ("consuls") qui sont des "fournisseurs négociants" de main-d'œuvre saisonnière auprès des producteurs de noisettes. Ils négocient à l'avance avec ces derniers la quantité de travailleurs à embaucher, le nombre de jours de travail et des logements. Avant la saison, soit ils se déplacent chez les producteurs pour traiter avec eux, soit ils les contactent par téléphone. Les elçi organisent aussi le voyage des travailleurs jusqu'au lieu de travail. Ce voyage se fait en dolmus ou en cars loués aux frais des travailleurs, qui sont placés chez les exploitants en équipe de 16 à 22 personnes selon les besoins.

On peut assimiler les elçi aux directeurs des agences de travail temporaire français qui fournissent de la main-d'œuvre aux entreprises privées. Sauf qu'en France, les agences d'intérim ont l'obligation de déclarer auprès dela Sécurité sociale tous les salariés travaillant sous leurresponsabilitéetdepayertoutesleurscotisations.
L'elçi kurde est plutôt proche du labor boss qui recrute des travailleurs mexicains pour les agriculteurs californiens ou du gatos, "chat" en brésilien, qui fournissait de la main-d'œuvre aux producteurs brésiliens de café dans les années 1980.

En principe, l'elçi kurde a des responsabilités à l'égard des travailleurs. Il se porte garant d'eux auprès des épiceries locales où ils s'approvisionnent en nourriture pendant la période de travail. À la fin du contrat, c'est l'elçi qui rembourse aux épiciers la dette de ses travailleurs en déduisant la somme de leur paie. Et c'est à lui que les agriculteurs payent les salaires des travailleurs. En cas de maladie et d'accident du travail ou de voiture, les elçi doivent accompagner les travailleurs à l'hôpital, ou, si cela est nécessaire, assurer leur rapatriement. En contrepartie, les travailleurs leur versent $10 \%$ de leur salaire. Les elçi travaillent en collaboration avec les çavus, qui sont responsables des équipes sur le lieu du travail. Les çavus sont pour la plupart des hommes, mais il y a aussi des femmes çavus. Il est important de noter qu’à la dif-

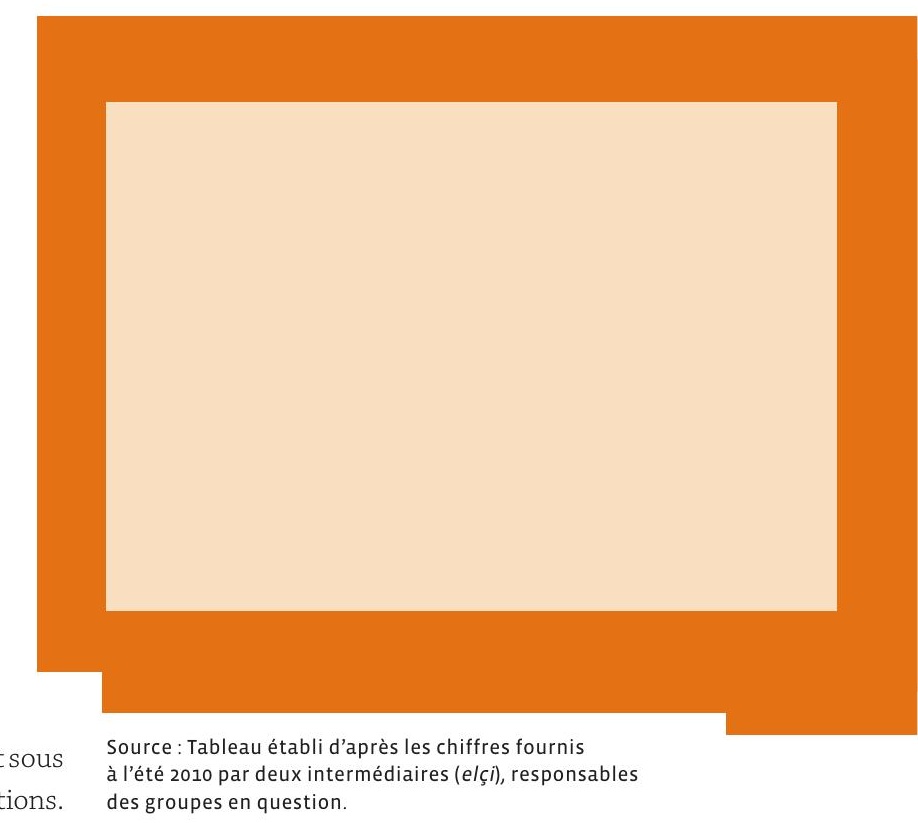


devrait rendre des comptes à leurs familles. Les çavus sont payés $50 \%$ (dans certains cas $100 \%$ de plus que les travailleurs. Ils versent aussi une commission de $10 \%$ à l'elçi. Afin de ne pas payer cette commission, certains çavus négocient directement avec des agriculteurs l'embauche des saisonniers pour l'année suivante. Toutefois, ils risquent de ne pas trouver assez de travail pour toute la saison. Or un ouvrier doit travailler au moins vingt jours pendant une saison pour amortir ses frais de voyage.

Chaque équipe comprend un ou deux porteurs (çuvalci) qui chargent les sacs remplis sur les remorques des tracteurs. Ils touchent le même salaire que les travailleurs. Chaque équipe a sa cuisinière qui prépare des repas (petit déjeuner, repas de midi et du soir) et fait le ménage du campement. Elle prépare aussi de l'eau chaude pour la douche des ouvriers et parfois lave à la main leurs vêtements. La cuisinière et les porteurs des sacs touchent le même salaire que les travailleurs et paient une commission de $10 \%$ à l'elçi.

Le travail des cuisinières est plus épuisant que celui des cueilleurs. Elles doivent aussi s'occuper de leurs enfants en bas âge qu'elles amènent avec elles sur leur lieu de travail.

Une partie des travailleurs saisonniers kurdes du département de Diyarbakir prennent le train pour se rendre aux départements de Düzce et de Sakarya où ils effectuent le ramassage des noisettes. Les travailleurs, venus avec toutes leurs affaires à la gare de Diyarbakir, attendent le train qui les transportera jusqu'à Sakarya, août 2009. (c) 2012. MUSTAfa AsLAN

férence des hommes, les femmes çavus participent aux tâches ménagères (ménage, vaisselle, lessive, etc.) dans le lieu de campement.

La responsabilité du çavus est d'assurer le bon déroulement du travail et de veiller à la rentabilité des ouvriers. Sans sa permission, les membres de l'équipe ne peuvent pas s'absenter du campement. Car, s'il leur arrivait quelque chose, c'est le çavus qui
Comme toute la famille part travailler dans les champs, il n'y a personne d'autre de confiance pour garder les enfants.

\section{Les conditions \\ de travail des travailleurs agricoles kurdes}

Les travailleurs commencent leur journée à 7 heures du matin et terminent à 18 heures, une journée aussi longue que celle des travailleurs marocains embauchés dans le sud de l'Espagne qui font "dix à quinze heures par jour avec un seul jour de 
repos dans la semaine ${ }^{16 "}$. En plus, ils doivent travailler sept jours sur sept. Dans la journée, ils ont droit à trois pauses : le matin 15 minutes, à midi une heure et l'après-midi encore 15 minutes.

En contrepartie de ces onze heures du travail, ils touchent 20 LT (13 \$ selon le cours de change à l'été 2009). Selon les informations que j'ai obtenues auprès des travailleurs et des agriculteurs rencontrés, les travailleurs turcs locaux faisant le même travail touchent entre 30 et 40 LT par jour pour huit heures de travail.

Les salaires sont fixés d'avance chaque année par une commission composée des seules autorités locales : la préfecture, la municipalité et la chambre des agriculteurs, sans que soient consultatés les elçi fournisseurs de main-d'œuvre. Cette procédure ressemble à celle appliquée en Californie où, pendant les années 1980, les employeurs recrutaient les travailleurs mexicains et vietnamiens en déterminant les salaires "unilatéralement et informellement ${ }^{17 "}$.

Un simple calcul de leurs dépenses et de leurs gains (voir tableau 2 ci-dessous) permet de constater que, pendant une saison qui dure en moyenne un mois et demi, les travailleurs saisonniers ne gagnent même pas l'équivalent du salaire minimum en vigueur en Turquie. De plus, près de la moitié de cet argent gagné sert à payer les frais de voyage et de nourriture, et à rembourser la commission de l'elçi.

Comme il n'y a pas de législation en Turquie pour les ouvriers agricoles permanents ou saisonniers ${ }^{18}$, la plupart des travailleurs rencontrés pendant l'enquête, conscients de leur situation, l'ont ainsi commentée : "Les chiens vivent mieux que nous!"

Pour se rendre sur le lieu du travail, ils effectuent entre seize et vingt-deux heures de voyage en dolmus ou en car dans des conditions dangereuses. Par exemple, plus de 20 travailleurs montent avec leurs affaires dans un dolmus dont la capacité maximale est de 14 places.

Une partie des travailleurs originaires de Siirt, Batman ou Diyarbakır voyagent en train, moyen de transport plus sûr et moins cher. Mais ils voyagent alors sous une chaleur torride et dans des trains bondés et souvent non climatisés ${ }^{19}$.

Leurs logements ressemblent aux "cortijo-chabola défoncés et rafistolés à grand renfort de plastique et carton" des ouvriers agricoles marocains travaillant dans la région d'Almeira, au sud de l'Espagne ${ }^{20}$. Il s'agit de logements vétustes, dépourvus de toute mesure d'hygiène, installés dans les champs de noisetiers. C'est à l'abri d'une bâche roulée autour de

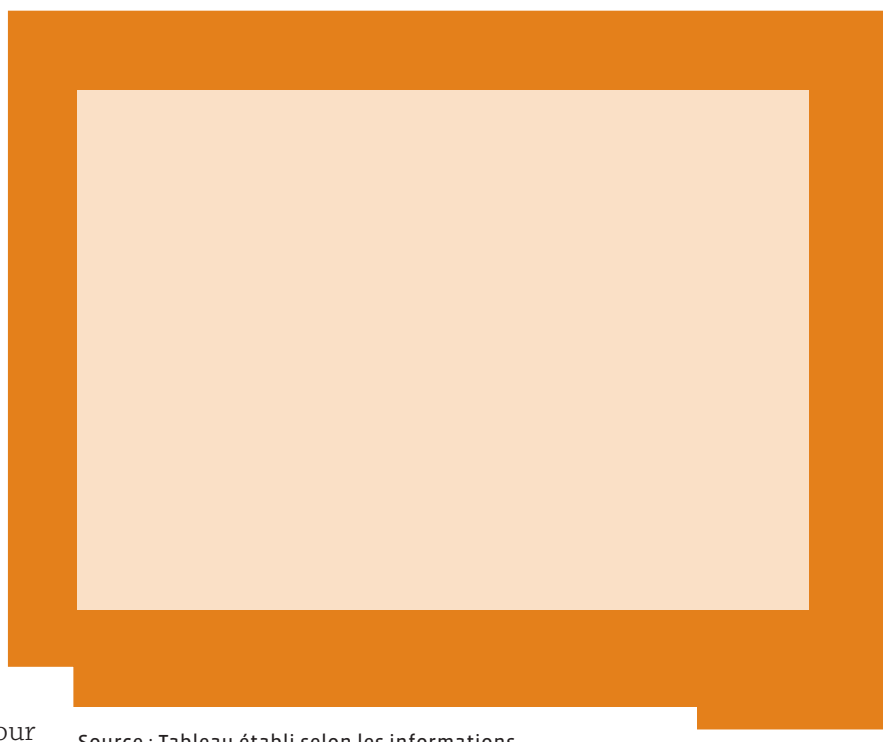

Source : Tableau établi selon les informations enregistrées auprès des ouvriers interrogés durant l'été de 2009.

quatre piquets en bois fixés au sol qu'ils prennent leur douche. Une fosse dont la profondeur dépasse à peine un mètre, couverte par quelques planches et close par une bâche fait office de W. C. Dans le meilleur des cas, les agriculteurs leur prêtent un 
entrepôt ou un hangar qu'ils aménagent avec les moyens du bord. S'ils osent protester sur leurs conditions de travail, la réaction des agriculteurs est immédiate : "Si cela ne vous convient pas, ne travaillez pas $^{21}$." Il y a en général de l'eau courante potable près du campement. Mais les équipes logées sous des bâches dans les champs ne l'ont pas toujours. Alors, les femmes de l'équipe vont la chercher à proximité, dans des jerricans portés sur l'épaule. Afin d'économiser, ces travailleurs sont obligés de se sous-alimenter. Pendant une saison de travail, ils mangent rarement de la viande ou des fruits, et ils consomment surtout du riz, du boulgour, des pâtes et des légumes. Pour le petit déjeuner, ils prennent des olives, du fromage et de la confiture. Malgré ces efforts, chaque ouvrier dépense en moyenne entre 100 et $150 \mathrm{LT}$ pour sa nourriture, ce qui représente la paie d'une semaine. Outre ces difficultés, ils sont confrontés à d'autres problèmes : les accidents du travail et de voyage, les ennuis de santé (il leur est difficile de se faire soigner dans les hôpitaux parce qu'ils n'ont pas de Sécurité sociale) et les discriminations ethnico-raciales que les représentants des institutions locales et les "autochtones" pratiquent à leur égard ${ }^{22}$. L'étude du développement de lagriculture intensive en Turquie et la situation économique de la région du sud-est de la Turquie, dont sont originaires les travailleurs saisonniers agricoles en question, permettent de mieux comprendre pourquoi ces saisonniers acceptent de travailler dans des conditions très difficiles : dépendance à l'égard du travail saisonnier, exploitation abusive par les elçi et les agriculteurs malveillants... La plupart étant sans profession et n'ayant pas d'autres choix que de chercher des travaux manuels, ils acceptent de travailler dans ces conditions avec un salaire journalier en dessous du Smic turc. En cas de contestation, les agriculteurs ou les elçi les renvoient en les remplaçant le lendemain par d'autres équipes prêtes à faire le même travail. Les travailleurs saisonniers agricoles kurdes sont quasiment les seuls à effectuer la cueillette des noisettes dans des conditions très proches du modèle de Huelva en Andalousie, avec toute une stratification interne : les étudiants, les femmes, les adolescent(e)s... Les travailleurs saisonniers agricoles kurdes, comme tous les travailleurs agricoles saisonniers, sont des laissés-pour-compte qui paient le tribut de la concurrence acharnée du marché agro-alimentaire mondial. Tant que le profit à tout prix et la concurrence du marché ne seront pas remis en cause, en Turquie, comme partout ailleurs, des salariés saisonniers seront contraints d'accepter les pires conditions de travail. 